\title{
Stability and Boundedness of Solutions to Nonautonomous Parabolic Integrodifferential Equations
}

\author{
Michael Gil' \\ Department of Mathematics, Ben Gurion University of the Negev, P.O. Box 653, 84105 Beer-Sheva, Israel \\ Correspondence should be addressed to Michael Gil'; gilmi@bezeqint.net
}

Received 20 January 2016; Revised 24 April 2016; Accepted 3 May 2016

Academic Editor: Quanxin Zhu

Copyright (C) 2016 Michael Gil'. This is an open access article distributed under the Creative Commons Attribution License, which permits unrestricted use, distribution, and reproduction in any medium, provided the original work is properly cited.

We consider a class of linear nonautonomous parabolic integrodifferential equations. We will assume that the coefficients are slowly varying in time. Conditions for the boundedness and stability of solutions to the considered equations are suggested. Our results are based on a combined usage of the recent norm estimates for operator functions and theory of equations on the tensor product of Hilbert spaces.

\section{Introduction and Statement of the Main Result}

This paper is devoted to stability and boundedness of solutions to parabolic integrodifferential equations, that is, equations containing the first derivative in time, integral operators, and partial derivatives in spatial variables. Such equations play an essential role in numerous applications, in particular, in the transport theory [1], continuous mechanics [2], and radiation theory [3]. For other applications see [4].

The literature on the asymptotic properties of integrodifferential equations is rather rich, but mainly ordinary (linear and nonlinear) equations, that is, equations without partial derivatives, were investigated; compare [5-9] and references given therein. For important stability results on stochastic partial differential equations see the papers [10-12].

The parabolic autonomous integrodifferential equations are investigated considerably less than the ordinary ones. For the recent papers on stability and the asymptotic behaviour of solutions to autonomous parabolic integrodifferential equations see [13-16] and references therein.

Despite many important applications the stability properties of solutions of nonautonomous integrodifferential equations have not been not investigated. The motivation of the present paper is to particularly fill a gap between the developed theory for ordinary integrodifferential equations and almost nonexistence theory for nonautonomous parabolic integrodifferential equations.
We obtain the main result of the paper for differentialoperator equation (1) in a Hilbert space. Based on that result we give explicit exponential stability conditions for the integrodifferential equations.

Let $H$ be a Hilbert space with a scalar product $(\cdot, \cdot)$, the norm $\|\cdot\|=\sqrt{(\cdot, \cdot)}$, and unit operator $I$. All the considered operators are assumed to be linear. For an operator $A, A^{*}$ is the adjoint one, $\sigma(A)$ is the spectrum, $\alpha(A)=\sup \operatorname{Re} \sigma(A)$, and $\operatorname{Dom}(A)$ denotes the domain.

Consider the equation

$$
\dot{u}=S u+B(t) u+F(t)
$$

$$
\left(u=u(t), t>0 ; \dot{u}=\frac{d u}{d t}\right),
$$

where $S$ is a closed constant operator in $H$ with a dense domain

$$
\begin{aligned}
\operatorname{Dom}(S) & =\operatorname{Dom}\left(S^{*}\right) \\
\gamma(S) & :=\sup _{w \in \operatorname{Dom}(S),\|w\|=1} \frac{\left(\left(S+S^{*}\right) w, w\right)}{2}<\infty ;
\end{aligned}
$$

$B(t)$ is an operator uniformly bounded on $[0, \infty)$, having a strong derivative uniformly bounded on $[0, \infty)$ and commuting with $S ; F(\cdot):[0, \infty) \rightarrow \operatorname{Dom}(S)$ satisfies the conditions pointed below. 
An important example of (1) is the boundary values problem

$$
\begin{aligned}
\frac{\partial u(t, x, y)}{\partial t}= & \frac{\partial^{2}}{\partial y^{2}} u(t, x, y)+c(t, x) u(t, x, y) \\
& +\int_{a}^{b} k(t, x, s) u(t, s, y) d s \\
& +f(t, x, y), \\
u(t, x, 0)= & u(t, x, 1)=0 \\
& (t>0 ; 0 \leq y \leq 1 ; a \leq x \leq b),
\end{aligned}
$$

where $c(\cdot, \cdot):[0, \infty) \times[a, b] \rightarrow \mathbb{R}, k(\cdot, \cdot, \cdot):[0, \infty) \times[a, b]^{2} \rightarrow$ $\mathbb{R}$ and $f(\cdot, \cdot):[0, \infty) \times[a, b] \times[0,1] \rightarrow \mathbb{R}$ are given functions and $u(\cdot, \cdot, \cdot)$ is unknown. In our reasonings below, instead of $[0,1]$ and $[a, b]$, one can consider closed bounded Euclidean sets and more general differential operators.

In the present paper we suggest the conditions providing stability and boundedness of solutions to (1) with slowly varying operator $B(t)$.

Certainly, (1) can be considered in some space as the equation $\dot{u}=A(t) u$ with an unbounded variable linear operator $A(t)$. This identification which is a common device in the theory of partial differential equations when passing from a parabolic equation to an abstract evolution equation turns out to be useful also here. Observe that $A(t)$ in the considered case has a special form: it is the sum of operators $S$ and $B(t)$. Besides, according to (3), $B(t)$ has a special structure. These facts enable us to use the information about the coefficients more completely than the theory of differential equations containing an arbitrary operator $A(t)$.

A solution to (1) for given $u_{0} \in \operatorname{Dom}(S)$ is a function $u:[0, \infty) \rightarrow \operatorname{Dom}(S)$ having a bounded measurable strong derivative and satisfying $u(0)=u_{0}$.

In particular, we will consider the homogeneous equation

$$
\dot{u}=S u+B(t) u \quad(t>0) .
$$

Equation (5) is said to be exponentially stable, if there are constants $M \geq 1, \epsilon>0$, such that $\|u(t)\| \leq$ $M \exp [-\epsilon t]\|u(0)\|(t \geq 0)$ for any solution of (1). Condition (2) implies that $S$ generates a strongly continuous semigroup $e^{S t}$; compare [17, Section I.4.4]. Since $B(t)$ is bounded and commutes with $S$ we can assert that (1) has solutions for any $u_{0} \in \operatorname{Dom}(S)$.

We will assume that, for each $\tau \geq 0$, the operator $B(\tau)+$ $\gamma(S) I$ is stable (Hurwitzian); namely,

$$
q(\tau):=2 \int_{0}^{\infty}\left\|e^{(B(\tau)+\gamma(S) I) s}\right\|^{2} d s<\infty .
$$

Now we are in a position to formulate the main result of this paper.

Theorem 1. Let conditions (2) and (6) and

$$
\sup _{t \geq 0} q^{2}(t)\left\|B^{\prime}(t)\right\|<2
$$

hold. Then (5) is exponentially stable. If, in addition, $\|F(t)\|$ is bounded and measurable on $[0, \infty)$, then any solution of $(1)$ is bounded on $[0, \infty)$.

\section{Proof of Theorem 1}

Put $C=S-\gamma(S) I$ and $A(t)=B(t)+\gamma(S) I$. Let $V(t)$ be the Cauchy operator to the equation

$$
\dot{v}(t)=A(t) v(t) \quad(t \geq 0) .
$$

That is, $V(t) v(0)=v(t)$ for any solution of (8). Taking

$$
u(t)=e^{C t} V(t) u_{0} \quad\left(u_{0} \in \operatorname{Dom}(S)\right)
$$

we have

$$
\dot{u}=(C+A(t)) u=(S+B(t)) u .
$$

Consequently, $e^{t C} V(t) u_{0}$ really is a solution to (5). Since

$$
\sup _{w \in \operatorname{Dom}(S),\|w\|=1}\left(\left(C+C^{*}\right) w, w\right) \leq 0
$$

we have $\left\|e^{t C}\right\| \leq 1, t \geq 0$; compare [17, Theorem I.4.2]. Thus, $\|u(t)\| \leq\left\|V(t) u_{0}\right\|$. So we have proved the following result.

Lemma 2. Under the hypothesis of Theorem 1, (5) is exponentially stable, provided (8) is exponentially stable.

Furthermore, recall that the equation

$$
A_{0}^{*} Y+Y A_{0}=E
$$

with given constant bounded stable operator $A_{0}$ (i.e., $\alpha\left(A_{0}\right)<0$ ) and a constant bounded operator $E$ has a solution $Y$ which is represented as

$$
Y=-\int_{0}^{\infty} e^{A_{0}^{*} s} E e^{A_{0} s} d s
$$

Compare [18, Section I.4.4]. Consequently, due to (2), the operator

$$
Q(t):=2 \int_{0}^{\infty} e^{A^{*}(t) s} e^{A(t) s} d s
$$

is a unique solution of the equation

$$
\begin{gathered}
A^{*}(t) Q(t)+Q(t) A(t)=-2 I \quad(t \geq 0), \\
\|Q(t)\| \leq q(t) .
\end{gathered}
$$

Lemma 3. Let condition (6) hold. Then $Q(t)$ is differentiable and $\left\|Q^{\prime}(t)\right\| \leq q^{2}(t)\left\|A^{\prime}(t)\right\|$.

Proof. Differentiating (15) we have

$$
\begin{aligned}
& A^{*}(t) Q^{\prime}(t)+Q^{\prime}(t) A(t) \\
& \quad=-\left(A^{*}(t)\right)^{\prime} Q(t)-Q(t) A^{\prime}(t) \quad(t \geq 0) .
\end{aligned}
$$


Hence, due to (15),

$$
\begin{aligned}
& Q^{\prime}(t) \\
& \quad=\int_{0}^{\infty} e^{A^{*}(t) s}\left(\left(A^{*}(t)\right)^{\prime} Q(t)+Q(t) A^{\prime}(t)\right) e^{A(t) s} d s .
\end{aligned}
$$

Thus,

$$
\begin{aligned}
\left\|Q^{\prime}(t)\right\| & \leq \frac{1}{2} q(t)\left\|\left(A^{*}(t)\right)^{\prime} Q(t)+Q(t) A^{\prime}(t)\right\| \\
& \leq q(t)\|Q(t)\|\left\|A^{\prime}(t)\right\| .
\end{aligned}
$$

Now (16) yields the result.

Lemma 4. Let

$$
\sup _{t \geq 0}\left\|Q^{\prime}(t)\right\|<2
$$

Then $(Q(t) u(t), u(t)) \leq(Q(0) u(0), u(0))(t \geq 0)$, for $a$ solution of (8).

Proof. Multiplying (8) by $Q(t)$ and doing the scalar product, we can write $\left(Q(t) u^{\prime}(t), u(t)\right)=(Q(t) A(t) u(t), u(t))$. Since

$$
\begin{aligned}
\frac{d}{d t}(Q(t) u(t), u(t))= & \left(Q(t) u^{\prime}(t), u(t)\right) \\
& +\left(u(t), Q(t) u^{\prime}(t)\right) \\
& +\left(Q^{\prime}(t) u(t), u(t)\right),
\end{aligned}
$$

it can be written as

$$
\begin{aligned}
\frac{d}{d t}(Q(t) u(t), u(t)) \\
=(Q(t) A(t) u(t), u(t))+(u(t), Q(t) A(t) u(t)) \\
\quad+\left(Q^{\prime}(t) u(t), u(t)\right) \\
=\left(\left(Q(t) A(t)+A^{*}(t) Q(t)\right) u(t), u(t)\right) \\
\quad+\left(Q^{\prime}(t) u(t), u(t)\right) \\
=-2(u(t), u(t))+\left(Q^{\prime}(t) u(t), u(t)\right) .
\end{aligned}
$$

Hence, condition (20) implies

$$
\begin{aligned}
\frac{d}{d t}(Q(t) u(t), u(t)) & \leq\left(-2+\left\|Q^{\prime}(t)\right\|\right)(u(t), u(t)) \\
& <0 .
\end{aligned}
$$

This proves the result.

Furthermore, for a stable operator $A_{0}$, put $y_{1}(t)=$ $e^{A_{0} t} v(v \in H)$. Then $\dot{y}_{1}=A_{0} y_{1}$, and

$$
\frac{d\left(y_{1}(t), y_{1}(t)\right)}{d t}=\left(\left(A_{0}+A_{0}^{*}\right) y_{1}(t), y_{1}(t)\right) \text {. }
$$

Hence

$$
\frac{d\left(y_{1}(t), y_{1}(t)\right)}{d t} \geq \lambda\left(A_{0}+A_{0}^{*}\right)\left(y_{1}(t), y_{1}(t)\right)
$$

and therefore

$$
\left\|e^{A_{0} t} v\right\|^{2} \geq e^{t \lambda\left(A_{0}+A_{0}^{*}\right)}\|v\|^{2}
$$

where $\lambda\left(A_{0}+A_{0}^{*}\right)$ is the smallest eigenvalue of $A_{0}+A_{0}^{*}$. Recall that $A_{0}$ is stable, so $\lambda\left(A_{0}+A_{0}^{*}\right)<0$. Then due to (13) and (26) with $E=-2 I$ we get

$$
\begin{aligned}
(Y h, h) & =2 \int_{0}^{\infty}\left(e^{A_{0}^{*} s} e^{A_{0} s} h, h\right) d s \\
& \geq 2 \int_{0}^{\infty} e^{\lambda\left(A_{0}+A_{0}^{*}\right) s} d s\|h\|^{2} \\
& =2\|h\|^{2}\left|\lambda\left(A_{0}+A_{0}^{*}\right)\right|^{-1} \quad(h \in H) .
\end{aligned}
$$

Hence, for any continuous function $u_{1}:[0, \infty) \rightarrow H$, we have

$$
\begin{aligned}
& \left(Q(t) u_{1}(t), u_{1}(t)\right) \\
& \quad \geq 2\left\|u_{1}(t)\right\|^{2}\left|\lambda\left(A(t)+A^{*}(t)\right)\right|^{-1} .
\end{aligned}
$$

Now the previous lemma implies

$$
\begin{array}{r}
(u(t), u(t)) \leq\left|\lambda\left(A(t)+A^{*}(t)\right)\right|(Q(0) u(0), u(0)) \\
(t \geq 0) .
\end{array}
$$

But $\left|\lambda\left(A(t)+A^{*}(t)\right)\right|$ is uniformly bounded and therefore all the solutions of (8) are uniformly bounded (i.e., (8) is Lyapunov stable). Furthermore, substitute into (8)

$$
u(t)=u_{\epsilon}(t) e^{-\epsilon t} \quad(\epsilon>0) .
$$

Then

$$
\dot{u}_{\epsilon}(t)=(A(t)+\epsilon I) u_{\epsilon}(t) .
$$

Applying our above arguments to (31) can assert that (31) with small enough $\epsilon>0$ is Lyapunov stable. So, due to (30), (8) is exponentially stable, provided (20) holds. Now Lemma 3 implies the following.

Lemma 5. Let $\sup _{t \geq 0} q^{2}(t)\left\|B^{\prime}(t)\right\|<2$. Then (8) is exponentially stable.

Proof of Theorem 1. The exponential stability of (5) immediately follows from Lemmas 2 and 5 , and the equality $A^{\prime}(t)=$ $B^{\prime}(t)$. The rest of the proof is obvious.

\section{Equations on a Tensor Product of Hilbert Spaces}

Let $E_{j}(j=1,2)$ be separable Hilbert spaces with scalar products $\langle\cdot, \cdot\rangle_{j}$, the unit operators $I_{j}$, and the norms $\|\cdot\|_{j}=$ 
$\sqrt{\langle\cdot, \cdot\rangle_{j}}$. Let $H=E_{1} \otimes E_{2}$ be the tensor product of $E_{1}$ and $E_{2}$. This means that $H$ is a collection of all formal sums of the form

$$
u=\sum_{j} y_{j} \otimes h_{j} \quad\left(y_{j} \in E_{1}, h_{j} \in E_{2}\right)
$$

with the understanding that

$$
\begin{aligned}
\lambda(y \otimes h) & =(\lambda y) \otimes h=y \otimes(\lambda h),\left(y+y_{1}\right) \otimes h \\
& =y \otimes h+y_{1} \otimes h, \\
y \otimes\left(h+h_{1}\right) & =y \otimes h+y \otimes h_{1} .
\end{aligned}
$$

Here $y, y_{1} \in E_{1} ; h, h_{1} \in E_{2}$, and $\lambda$ is a number. The scalar product in $H$ is defined by

$$
\begin{aligned}
\left(y \otimes h, y_{1} \otimes h_{1}\right)_{H}= & \left(y \otimes h, y_{1} \otimes h_{1}\right) \\
= & \left\langle y, y_{1}\right\rangle_{1}\left\langle h, h_{1}\right\rangle_{2} \\
& \quad\left(y, y_{1} \in E_{1}, h, h_{1} \in E_{2}\right)
\end{aligned}
$$

and the norm $\|\cdot\| \equiv\|\cdot\|_{H}:=\sqrt{(\cdot, \cdot)_{H}}$. The closure of $H$ in the norm $\|\cdot\|_{H}$ is a Hilbert space; compare [19]. It is again denoted by $H$. In addition, the unit operator $I=I_{H}$ in $H$ equals $I_{1} \otimes I_{2}$.

Furthermore, for a Hilbert-Schmidt operator $C$ in $E_{1}, N_{2}(C)$ denotes the Hilbert-Schmidt norm: $N_{2}(C)=$ $\sqrt{\text { trace } C C^{*}}$. Let $S_{0}$ be a closed constant operator in $E_{2}$ with a dense domain

$$
\begin{aligned}
\operatorname{Dom}\left(S_{0}\right) & =\operatorname{Dom}\left(S_{0}^{*}\right), \\
\gamma\left(S_{0}\right) & :=\sup _{w \in \operatorname{Dom}\left(S_{0}\right),\|w\|=1} \frac{\left(\left(S_{0}+S_{0}^{*}\right) w, w\right)}{2}<\infty .
\end{aligned}
$$

Let $B_{0}(t)$ be a linear operator in $E_{1}$ uniformly bounded on $[0, \infty)$, having a strong derivative uniformly bounded on $[0, \infty)$. Put $B(t)=B_{0}(t) \otimes I_{2}, S=I_{1} \otimes S_{0}$ and assume that, for each $\tau \geq 0$, the operator $B_{0}(\tau)+\gamma\left(S_{0}\right) I_{1}$ is stable:

$$
q_{0}(\tau):=2 \int_{0}^{\infty}\left\|e^{\left(B_{0}(\tau)+\gamma\left(S_{0}\right) I_{1}\right) s}\right\|^{2} d s<\infty \quad(\tau \geq 0) .
$$

Let

$$
\sup _{t \geq 0} q_{0}^{2}(t)\left\|B_{0}^{\prime}(t)\right\|<2 .
$$

Then condition (7) holds and, therefore, (5) is exponentially stable. If, in addition, $\|F(t)\|$ is bounded on $[0, \infty)$, then any solution of $(1)$ is bounded on $[0, \infty)$.

Furthermore, from (36), it follows that $\alpha\left(B_{0}(\tau)\right)+\gamma\left(S_{0}\right)<$ 0 . Assume that

$$
B_{0}(t)-B_{0}^{*}(t) \text { is a Hilbert-Schmidt operator }
$$

and put

$$
\begin{array}{r}
g(t):=\left(\frac{1}{2} N_{2}^{2}\left(B_{0}(t)-B_{0}^{*}(t)\right)\right. \\
\left.-2 \sum_{k=1}^{\infty}\left|\operatorname{Im} \lambda_{k}\left(B_{0}(t)\right)\right|^{2}\right)^{1 / 2},
\end{array}
$$

where $\lambda_{k}\left(B_{0}(t)\right)$ are nonreal eigenvalues of $B_{0}(t)$ taken with their multiplicities. Clearly,

$$
g(t) \leq \frac{N_{2}\left(B_{0}(t)-B_{0}^{*}(t)\right)}{\sqrt{2}} .
$$

Due to [20, Example 7.10.3],

$$
\left\|e^{B_{0}(\tau) t}\right\| \leq \exp \left[\alpha\left(B_{0}(\tau)\right) t\right] \sum_{k=0}^{\infty} \frac{g^{k}(\tau) t^{k}}{(k !)^{3 / 2}} \quad(\tau, t \geq 0) .
$$

So

$$
\begin{aligned}
q_{0}(\tau) \leq 2 \int_{0}^{\infty} \exp \left[2\left(\alpha\left(B_{0}(\tau)+\gamma\left(S_{0}\right)\right)\right) t\right] \\
\cdot\left(\sum_{k=0}^{\infty} \frac{g^{k}(\tau) t^{k}}{(k !)^{3 / 2}}\right)^{2} d t \\
=2 \int_{0}^{\infty} \exp \left[2\left(\alpha\left(B_{0}(\tau)+\gamma\left(S_{0}\right)\right)\right) t\right] \\
. \sum_{j, k=0}^{\infty} \frac{(g(\tau) t)^{k+j}}{(j ! k !)^{3 / 2}} d t .
\end{aligned}
$$

Hence $q_{0}(\tau) \leq \psi\left(\tau, \gamma\left(S_{0}\right)\right)$, where

$$
\begin{aligned}
& \psi\left(\tau, \gamma\left(S_{0}\right)\right) \\
& \quad:=2 \sum_{j, k=0}^{\infty} \frac{(k+j) ! g^{j+k}(\tau)}{\left(2\left|\alpha\left(B_{0}(\tau)\right)+\gamma\left(S_{0}\right)\right|\right)^{j+k+1}(j ! k !)^{3 / 2}} .
\end{aligned}
$$

This inequality, (37), and Theorem 1 imply the following.

Corollary 6. Let conditions (35) and

$$
\sup _{t \geq 0} \psi^{2}\left(t, \gamma\left(S_{0}\right)\right)\left\|B_{0}^{\prime}(t)\right\|<2
$$

hold. Then (5) is exponentially stable.

\section{Example}

Put $\Omega=[a, b] \times[0,1]$. In this section $E_{1}=L^{2}(a, b), E_{2}=$ $L^{2}(0,1)$, and $H=E_{1} \otimes E_{2}=L^{2}(\Omega)$ are the Hilbert spaces of real functions with usual scalar products.

Consider problem (3), (4), assuming that, for almost all $x, s \in[0,1], c(t, x)$ and $k(t, x, s)$ have bounded measurable derivatives $c_{t}^{\prime}(t, x)$ and $k_{t}^{\prime}(t, x, s)$. In addition, the operators $B_{0}(t)$ and $B_{0}^{\prime}(t)$ defined in $L^{2}(a, b)$ by

$$
\begin{array}{r}
B_{0}(t) w(x)=c(t, x) w(x)+\int_{a}^{b} k(t, x, s) w(s) d s, \\
B_{0}^{\prime}(t) w(x)=c_{t}^{\prime}(t, x) w(x)+\int_{a}^{b} k_{t}^{\prime}(t, x, s) w(s) d s \\
\left(x \in[a, b] ; w \in L^{2}(a, b)\right),
\end{array}
$$


respectively, are assumed to be bounded uniformly in $t \in$ $[0, \infty)$. In addition,

$$
\begin{aligned}
\hat{g}(t) & =\sqrt{\frac{1}{2}} N_{2}\left(B(t)-B_{0}^{*}(t)\right) \\
& =\sqrt{\frac{1}{2}}\left(\iint_{a}^{b}(k(t, x, s)-k(t, s, x))^{2} d s d x\right)^{1 / 2} \\
& <\infty .
\end{aligned}
$$

Let $S_{0}=d^{2} / d x^{2}$ with the domain

$$
\begin{gathered}
\operatorname{Dom}\left(S_{0}\right)=\left\{w \in L^{2}(0,1): \frac{d^{2} w(x)}{d x^{2}}\right. \\
\left.\in L^{2}(0,1) ; w(0)=w(1)=0\right\} .
\end{gathered}
$$

Then $S_{0}$ is self-adjoint with the eigenvalues $-\pi k^{2}(k=$ $1,2, \ldots)$. So $\gamma\left(\mathrm{S}_{0}\right)=-\pi^{2}$. Assume that

$$
\sup _{t \geq 0} \alpha\left(B_{0}(t)\right)-\pi^{2}<0
$$

and put

$$
\psi_{\pi}(\tau):=2 \sum_{j, k=0}^{\infty} \frac{(k+j) ! \hat{g}^{j+k}(\tau)}{\left(2\left|\alpha\left(B_{0}(\tau)\right)-\pi^{2}\right|\right)^{j+k+1}(j ! k !)^{3 / 2}} .
$$

Now Corollary 6 implies the following.

Corollary 7. Let conditions (48) and

$$
\sup _{t \geq 0}^{2} \psi_{\pi}^{2}(t)\left\|B_{0}^{\prime}(t)\right\|<2
$$

hold. Then the equation

$$
\begin{aligned}
\frac{\partial u(t, x, y)}{\partial t}= & \frac{\partial^{2}}{\partial y^{2}} u(t, x, y)+c(t, x) u(t, x, y) \\
& +\int_{a}^{b} k(t, x, s) u(t, s, y) d s
\end{aligned}
$$

with condition (4) is exponentially stable. If, in addition, $f_{y y}^{\prime \prime}(t, \cdot, \cdot) \in L^{2}(\Omega)$ and $\|f(t, \cdot, \cdot)\|_{L^{2}(\Omega)}$ is uniformly bounded on $[0, \infty)$, then any solution of (3) is uniformly bounded on $[0, \infty)$ in the norm of $L^{2}(\Omega)$.

To estimate $\alpha\left(B_{0}(t)\right)$ one can apply various bounds for spectra of integral operators. For instance, consider the equation

$$
\begin{aligned}
\frac{\partial u(t, x, y)}{\partial t}= & \frac{\partial^{2}}{\partial y^{2}} u(t, x, y)+c(t, x) u(t, x, y) \\
& +\int_{a}^{x} k(t, x, s) u(t, s, y) d s
\end{aligned}
$$

with condition (4). So $B_{0}(t) w(x)=c(t, x) w(x)+(V(t) w)(x)$, where

$$
\begin{aligned}
(V(t) w)(x)=\int_{a}^{x} k(t, x, s) w(s) d s & \\
& \left(x \in[a, b] ; w \in L^{2}(a, b)\right) .
\end{aligned}
$$

It is simple to check that in this case $g(t)=N_{2}(V(t))$ and $\sigma\left(B_{0}(t)\right)=\{c(t, x): x \in[a, b]\}$. Thus $\alpha\left(B_{0}(t)\right)=$ $\sup _{x \in[0,1]} c(x, t)$ and

$$
\begin{aligned}
& \psi_{\pi}(\tau) \\
& \quad:=2 \sum_{j, k=0}^{\infty} \frac{(k+j) ! N_{2}^{j+k}(V(\tau))}{\left(2\left|\sup _{x \in[0,1]} c(x, \tau)-\pi^{2}\right|\right)^{j+k+1}(j ! k !)^{3 / 2}},
\end{aligned}
$$

provided

$$
\sup _{x \in[0,1]} c(x, t)-\pi^{2}<0 .
$$

Now we can directly apply the previous corollary. The general case of (51) can be considered as a perturbation of (52).

\section{Conclusion}

We have established the explicit stability test for linear parabolic integrodifferential equations in the case of slow varying in time coefficients. Stability of such equations has not been investigated in the available literature. As the example shows, the test is simply applicable and enables us to avoid the construction of the Lyapunov functionals in appropriate situations.

\section{Competing Interests}

The author declares that they have no competing interests.

\section{References}

[1] H. G. Kaper, C. G. Lekkerkerker, and J. Hejtmanek, Spectral Methods in Linear Transport Theory, Birkhäuser, Basel, Switzerland, 1982.

[2] V. M. Aleksandrov and E. V. Kovalenko, Problems in Continuous Mechanics with Mixed Boundary Conditions, Nauka, Moscow, Russia, 1986 (Russian).

[3] G. I. Marchuk, The Methods of Calculation for Nuclear Reactors, Atomizdat, Moscow, Russia, 1961 (Russian).

[4] J. Appel, A. Kalitvin, and P. Zabreiko, Partial Integral Operators and Integrodifferential Equations, Marcel Dekker, New York, NY, USA, 2000.

[5] R. P. Agarwal, A. Domoshnitsky, and Y. Goltser, "Stability of partial functional integro-differential equations," Journal of Dynamical and Control Systems, vol. 12, no. 1, pp. 1-31, 2006.

[6] A. Domoshnitsky and Ya. M. Goltser, "Approach to study of bifurcations and stability of integro-differential equations," Mathematical and Computer Modelling, vol. 36, no. 6, pp. 663678, 2002. 
[7] A. D. Drozdov and M. I. Gil', "Stability of linear integrodifferential equations with periodic coefficients," Quarterly Journal of Mechanics and Applied Mathematics, vol. 54, no. 4, pp. 609-624, 1996.

[8] M. I. Gil, "'On stability of linear Barbashin type integrodifferential equations," Mathematical Problems in Engineering, vol. 2015, Article ID 962565, 5 pages, 2015.

[9] Y. Goltser and A. Domoshnitsky, "Bifurcations and stability of integro-differential equations," Nonlinear Analysis: Theory, Methods \& Applications, vol. 47, no. 2, pp. 953-967, 2001.

[10] X. Yang and Q. Zhu, "Existence, uniqueness, and stability of stochastic neutral functional differential equations of Sobolevtype," Journal of Mathematical Physics, vol. 56, no. 12, Article ID 122701, 16 pages, 2015.

[11] X. Yang and Q. Zhu, " $p$-th moment exponential stability of stochastic partial differential equations with Poisson jumps," Asian Journal of Control, vol. 16, no. 5, pp. 1482-1491, 2014.

[12] Q. Zhu, "Asymptotic stability in the p-th moment for stochastic differential equations with Levy noise," Journal of Mathematical Analysis and Applications, vol. 416, no. 1, pp. 126-142, 2014.

[13] N. M. Chuong, T. D. Ke, and N. N. Quan, "Stability for a class of fractional partial integro-differential equations," Journal of Integral Equations and Applications, vol. 26, no. 2, pp. 145-170, 2014.

[14] J. Cao and Z. Huang, "Existence and exponential stability of weighted pseudo almost periodic classical solutions of integrodifferential equations with analytic semigroups," Differential Equations and Dynamical Systems, vol. 23, no. 3, pp. 241-256, 2015.

[15] H. Matsunaga and H. Hashimoto, "Asymptotic stability and stability switches in a linear integro-differential system," Differential Equations \& Applications, vol. 3, no. 1, pp. 43-55, 2011.

[16] N. T. Dung, "On exponential stability of linear Levin-Nohel integro-differential equations," Journal of Mathematical Physics, vol. 56, no. 2, Article ID 022702, 10 pages, 2015.

[17] S. G. Krein, Linear Differential Equations in Banach Space, AMS, Providence, RI, USA, 1971.

[18] Y. L. Daleckii and M. G. Krein, Stability of Solutions of Differential Equations in Banach Space, American Mathematical Society, Providence, RI, USA, 1974.

[19] N. Dunford and J. T. Schwartz, Linear Operators, Part I, Interscience, New York, NY, USA, 1963.

[20] M. I. Gil', Operator Functions and Localization of Spectra, vol. 1830 of Lecture Notes in Mathematics, Springer, Berlin, Germany, 2003. 


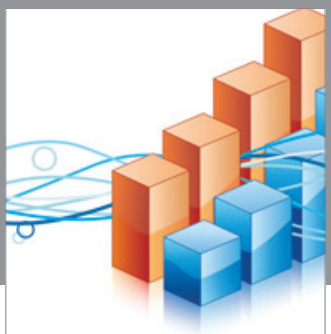

Advances in

Operations Research

vatem alat4

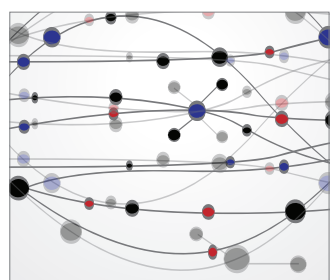

\section{The Scientific} World Journal
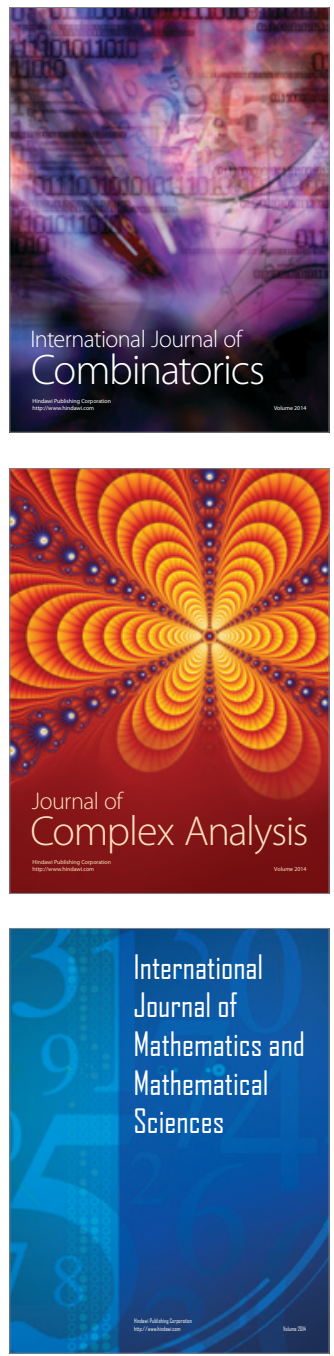
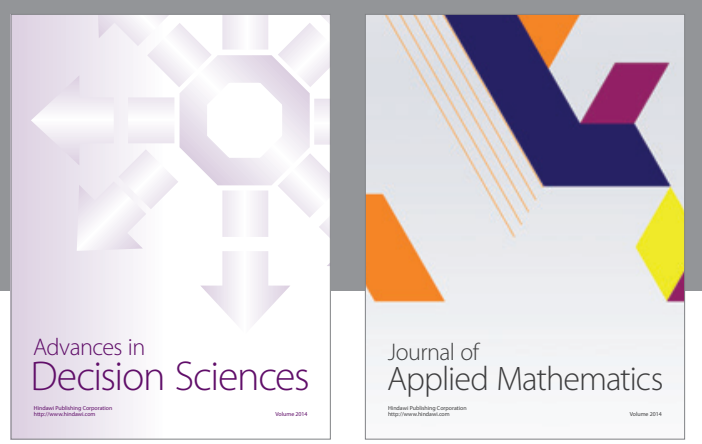

Algebra

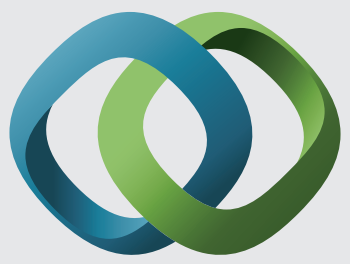

\section{Hindawi}

Submit your manuscripts at

http://www.hindawi.com
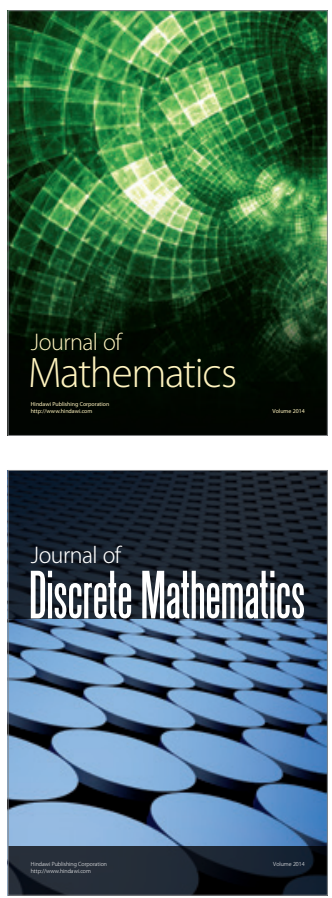

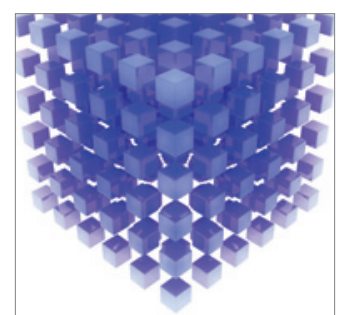

Mathematical Problems in Engineering
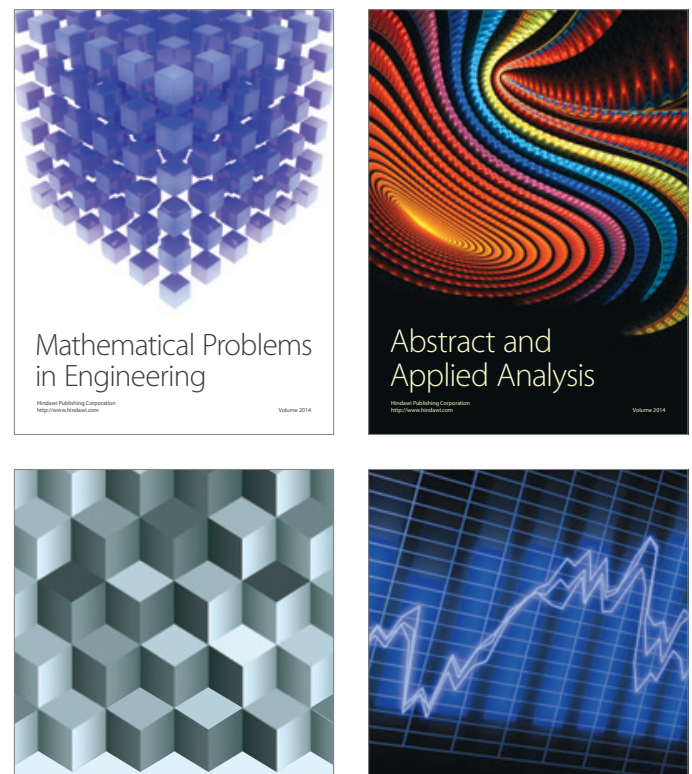

Journal of

Function Spaces

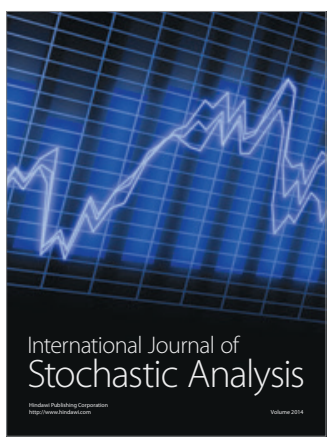

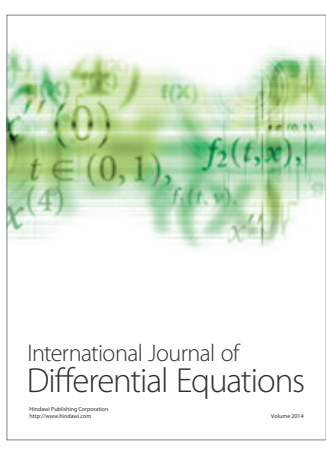
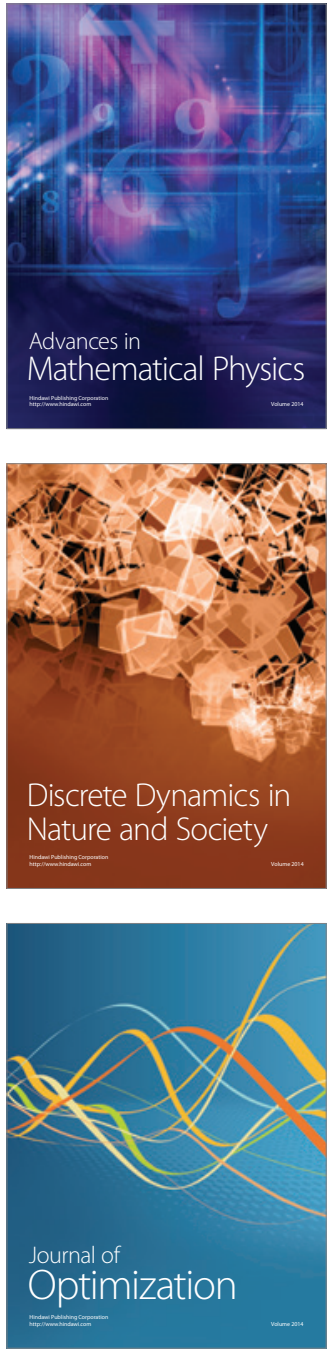